\title{
Objectively Monitored Physical Activity and Sitting Time in Bariatric Patients Pre- and Post-Surgery
}

Keywords: Physic al activity; Sedentary behaviour; Bariatric surgery; Obesity; Activity monitoring

\begin{abstract}
Purpose: Sedentary time, or prolonged sitting/lying time, is an emerging risk factor for severe obesity, independent of physical activity (PA). The purpose of this study was to characterize a mbulatory PA as well as sitting/lying time using accelerometry before and after bariatric surgery.

Material and methods: Seventeen partic ipants (58.5\% female) with a mean age of $46.5 \pm 10.1$ years, and a Body Mass Index (BMI) of $48.8 \pm$ $6.2 \mathrm{~kg} / \mathrm{m}^{2} \mathrm{scheduled}$ for bariatric surgery were included in the a nalysis. ActivPAL ${ }^{\text {IM }}$ tri-axial accelerometers were attached to the participants' mid-thigh and wom for 24 hours a day for up to seven consecutive days before surgery, and at three, and six months following bariatric surgery. Mean steps, transitions from sitting-to-standing, as well as standing, walking and sitting/lying time per 24 hours were measured.

Results: Prior to bariatric surgery, partic ipants spent $18.6 \pm 1.5$ hours per 24 hours sitting/lying, representing over $75 \%$ of their day. Total sitting/lying time per 24 hours did not change at three or six months after surgery. Baseline accumulated steps per day averaged $6139 \pm$ 2720 and did not change over time.

Conclusions: Given that PA and sitting/lying time per 24 hours a re believed to independently contribute to energy balance and health outcomes, clinicians and health professionals should intervene more rigorously to simultaneously reduce daily sitting time and increase levels of PA to help bariatric patients suc cessfully manage their weight over the long-term post-surgery.
\end{abstract}

\section{Abbreviations}

PA: Physical Activity

\section{Introduction/ Purpose}

An active lifestyle is one of the strongest predictors of long-term weight control and is associated with improved health outcomes [1]. Sedentary behavior refers to a sitting or reclining posture during commuting, in the workplace, during home and leisure time, and is an emerging risk factor for obesity and cardiovascular disease that is independent of levels of physical activity (PA) [2-5]. Furthermore, severely obese individuals and bariatric surgery candidates seem to report higher levels of sedentary behavior and are less likely to acquire adequate amounts of daily PA compared to the general population $[6,7]$.

A recent review of bariatric candidates awaiting surgery found that they spent $81 \%$ of their waking hours engaged in sedentary behaviors, $26 \%$ more than the average American adult [8,9]. While various levels of PA have been reported pre-operatively, overall it seems that bariatric patients remain in an inactive state following
Journal of

Obesity and

Bariatrics

\section{Olivier Babineau ${ }^{1}$, Tamara E Carver ${ }^{1}$, Ryan ER Reid $^{1 *}$, Nicolas V Christou ${ }^{2}$ and Ross E Andersen ${ }^{1}$}

${ }^{1}$ Department of Kinesiology and Physical Education, McGill University, Montreal, QC, Canada

${ }^{2}$ Bariatric Surgery, McGill University Health Center, Montreal, QC, Canada

\section{*Address for Correspondence}

Ryan ER Reid, BSc, Department of Kinesiology and Physical Education, McGill University, 475 avenue des Pins Ouest, Montreal, QC H2W 1S4, Canada, Tel: (514) 578-1813; E-mail: ryan.reid@mail.mcgill.ca

\section{Submission: 06 August 2015}

Accepted: 12 August 2015

Published: 17 August 2015

Copyright: (๑) 2015 Babineau O, et al. This is an open access article distributed under the Creative Commons Attribution License, which permits unrestricted use, distribution, and reproduction in any medium, provided the original work is properly cited.

Reviewed \& Approved by: Dr. Francesco Saverio Papadia, Assistant Professor of Surgery, University of Genoa School of Medicine, Italy

surgery $[10,11]$. A recent review found that self-reported levels of PA increased after surgery, however the authors stressed that objectively measured PA is required to better understand the PA and sedentary patterns for this population $[12,13]$. To the best of our knowledge, no studies have simultaneously examined objectively determined PA levels, sitting/lying time, and transitions from sitting-to-standing in bariatric surgery patients from pre- to post-surgery.

The primary aim of this study was to objectively monitor, with the use of wearable accelerometry, ambulatory PA and sitting/lying time per 24 hours before and after bariatric surgery. In addition, the secondary aim was to evaluate associations between sitting/lying time per 24 hours, daily steps, and transitions from sitting-to-standing to changes in body composition obtained from dual energy $\mathrm{x}$-ray absorptiometry (DXA).

\section{Materials and Methods}

Participants were recruited from the McGill University Health Center, Montreal, Québec. All participants had been medically cleared for surgery and were recruited from orientation meetings where they received information about study objectives and protocols. Only participants who were ambulatory were eligible to participate in this trial. After having shown interest, patients who met the eligibility criteria for bariatric surgery were approached by the study coordinator [14]. A total of 34 patients were consented, assessed at baseline, and underwent surgery. The number of participants included in the analysis for this study was 17. Exclusion from analysis occurred due to failed recordings with the ActivPAL ${ }^{\mathrm{TM}}$ in 13 participants at the three-month, and 17 participants at the sixmonth post-surgical follow-ups. All participants underwent rouxen-y gastric bypass surgery. The protocol was reviewed and approved by the Institutional Review Board of McGill University and all participants provided informed consent. 
Citation: Babineau O, Carver TE, Reid RER, Christou NV, Andersen RE. Objectively Monitored Physical Activity and Sitting Time in Bariatric Patients Pre- and Post-Surgery. J Obes Bariatrics. 2015;2(2): 5.

Height and weight were measured using a wall-mounted stadiometer and a bariatric scale $\left(\mathrm{SECA}^{\mathrm{TM}}\right.$ Medical Scales and Measuring Devices, U.S.) respectively. Body composition was assessed using the Lunar iDXA ${ }^{\mathrm{TM}}$ (GE Healthcare ${ }^{\mathrm{TM}}$, USA) whole body composition scanner previously described [15].

Objective assessment of PA and sitting/lying time was obtained using a wearable tri-axial accelerometer known as the ActivPAL ${ }^{\mathrm{TM}}$ (PAL Technologies Ltd., Glasgow, UK). This device detects limb position using an inclinometer that samples at $10 \mathrm{~Hz}$ and provides output in 15 second epochs. This unobtrusive activity monitor has been validated and is reliable to evaluate steps/day (a strong indicator of physical activity and successful long-term weight loss [1]), time spent in sitting/lying behaviors (an emerging risk factor for obesity and cardiovascular disease that is independent of levels of PA [2-5]), sit-to-stand transitions (a marker of bouts of sedentary time [16]), standing, and walking time [17,18]. During the initial baseline visit, the ActivPAL ${ }^{\mathrm{TM}}$ was placed inside a finger cot and affixed on the participant's mid-thigh using a Tegaderm ${ }^{\mathrm{TM}}$ adhesive patch. Participants were asked to remove the unit only upon bathing or undertaking any other prolonged underwater-related activity. Following the seven-day wear period, the unit was returned by mail and the participants' data uploaded through ActivPAL ${ }^{\mathrm{TM}}$ v6.3.4 software. A valid wear period was defined as a minimum of three days with at least one weekend-day. The height, weight, body composition, and ActivPAL ${ }^{\mathrm{TM}}$ measures were assessed again at three, and six months after surgery. All accelerometer measures were examined for both weekdays and weekends for both evenings (6:00 PM to 12:00 AM, commonly an adult's weekday leisure time), and the total 24 hours/ day. For this analysis, sitting/lying time per 24 hours was used instead of daily sedentary time as sleeping logs were not used during the initial data collection period. Without a self-monitored sleep log, the ActivPAL $^{\mathrm{TM}}$ is not capable of distinguishing between daily sedentary time and total sitting/lying time (PAL Technologies Ltd., Glasgow, UK).

Longitudinal changes in sitting/lying time per 24 hours, steps/day and sit-to-stand transitions were examined using a repeated measure analysis of covariance (ANCOVA), with adjusted means reported controlling for age and seasons. Relationships between variables of interest were assessed using Pearson's correlation coefficient. Alpha was set at $\mathrm{p}<0.05$ and all analyses were conducted using $\mathrm{IBM}^{\mathrm{TM}}$ SPSS version 20 . In order to identify a clinically meaningful change in sitting/lying time per 24 hours and PA, a modest Effect Size of 0.6 was used, alpha was set at 0.05 , and $\beta$ at 0.80 using a repeated measures design with three assessment points [19]. Along with previous findings, we estimated that with 17 participants, a clinically meaningful reduction in sitting/lying time per 24 hours of 1 hour/ day, and increases of 1600 steps/day with 10 sit-to-stand transitions would be required.

\section{Results}

Seventeen severely obese individuals (ten female and seven male) with a mean age of $46.5 \pm 10.1$ years, BMI of $48.8 \pm 6.2 \mathrm{~kg} / \mathrm{m}^{2}$, height of $168.7 \pm 0.1 \mathrm{~cm}$, total body weight of $140 \pm 26.4 \mathrm{~kg}$, and percent body fat of $50.5 \pm 4.8 \%$, were included in the ANCOVA. Weight related measures are presented in Table 1 in addition to their changes at month's three and six post-surgery. Weight was reduced significantly at three-months $(\mathrm{p}<0.001)$ and continued to decline at six-months $(\mathrm{p}<0.001)$ after surgery.

Participants wore the ActivPAL ${ }^{\mathrm{TM}}$ across all time points on average $5.2 \pm 0.7$ days, including $3.3 \pm 0.7$ days during the week and $1.9 \pm 0.3$ on weekend days. Participants' average sitting/lying time per 24 hours, sit-to-stand transitions, standing time, walking time, and steps/day are presented in Table 2.

As seen in Table 2, sitting/lying time per 24 hours demonstrated no change from baseline to three $(\mathrm{p}=0.969)$ and six-months $(\mathrm{p}=$ 0.308 ) post-surgery. Age and season-adjusted total steps/day averaged $6139 \pm 2720$ and did not change over the six months post-surgery (Table 2). Prior to surgery, $41 \%$ of participants accumulated below the inactive lifestyle threshold of $<5000$ steps/day, $29 \%$ were in the low-active category (5000-7500 steps/day), while $24 \%$ were classified as somewhat active (7500-9999 steps/day), and $6 \%$ as active ( $\geq 10000$ steps/day) [20]. Post-surgery, no differences were observed in the step count activity classification categories mentioned above (Figure 1). At baseline, steps/day during the week and on weekends was similar, averaging $6213 \pm 2732$ and $6059 \pm 3189$ steps/day respectively $(\mathrm{p}=$ 0.796). This pattern of activity did not change at three- $(\mathrm{p}=0.237)$, or six-months $(\mathrm{p}=0.733)$ after surgery. In addition, the number of steps accumulated during evening leisure time averaged $1206 \pm 532$ at baseline, representing $22 \%$ of the total daily steps taken, which also did not change following surgery (Table 2).

Participants spent $18.6 \pm 1.5$ hours per 24 hours in sitting/lying positions, representing over $75 \%$ of their day. In addition, sitting/ lying time per 24 hours did not differ between the week and weekend at baseline $(\mathrm{p}=0.926)$, three-months $(\mathrm{p}=0.621)$, or six-months $(\mathrm{p}=$ 0.449 ) after surgery respectively. Changes in daily and evening leisure sitting/lying times at baseline, three, and six-months are displayed in Table 2.

Transitions accumulated per day did not change over time, as participants before surgery made $54.8 \pm 19.7$ transitions per day, and at three, and six-months after surgery averaged $49.1 \pm 14.3$ and 49.6 \pm 12.6 transitions per day respectively. In addition, no changes in sitto-stand transitions were observed between weekdays or weekends before surgery $(\mathrm{p}=0.125)$, at three $(\mathrm{p}=0.672)$, or six- $(\mathrm{p}=0.246)$ months after surgery.

No relationships were observed between body fat percentage and changes in steps/day or overall sitting/lying time (all $\mathrm{r}$ values $<0.20$ ) at either three- or six-months after surgery. While the number of sit-

Table 1: Weight and body composition related variables before and after bariatric surgery.

\begin{tabular}{|l|c|c|c|}
\hline \multicolumn{1}{|c|}{ Parameter } & \multirow{2}{*}{ Baseline } & \multicolumn{2}{c|}{ Postoperatively } \\
\cline { 3 - 4 } & & $\mathbf{3}$ Months & $\mathbf{6}$ Months \\
\hline Body Weight $(\mathrm{kg})$ & $140.0 \pm 26.4$ & $110.0 \pm 24.4^{*}$ & $99.7 \pm 26.1^{*} \dagger$ \\
\hline BMI $\left(\mathrm{kg} / \mathrm{m}^{2}\right)$ & $48.8 \pm 6.2$ & $38.4 \pm 6.8^{*}$ & $34.8 \pm 7.5^{\star} \dagger$ \\
\hline Percent Body Fat (\%) & $50.5 \pm 4.8 \ddagger$ & $46.4 \pm 6.0^{*}$ & $41.1 \pm 7.0^{*} \dagger$ \\
\hline Weight Loss $(\mathrm{kg})$ & - & $30.2 \pm 9.8$ & $40.3 \pm 13.0 \dagger$ \\
\hline Weight Loss $(\%)$ & - & $21.6 \pm 6.1$ & $29.1 \pm 8.8 \dagger$ \\
\hline
\end{tabular}

Data presented as mean $\pm S D,{ }^{*} p<0.05$ from baseline, $\dagger p<0.05$ from 3 months to 6 months,

$\ddagger \mathrm{n}=15$, Two patients exceeded weight limit for iDXA at baseline.

BMI: Body Mass Index 
Citation: Babineau O, Carver TE, Reid RER, Christou NV, Andersen RE. Objectively Monitored Physical Activity and Sitting Time in Bariatric Patients Pre- and Post-Surgery. J Obes Bariatrics. 2015;2(2): 5.

ISSN: 2377-9284

Table 2: Bariatric surgery patients daily steps and time-spent sitting/lying across time.

\begin{tabular}{|c|c|c|c|}
\hline \multirow{2}{*}{ Parameters } & \multirow{2}{*}{ Baseline } & \multicolumn{2}{|c|}{ Postoperatively } \\
\hline & & 3 Months & 6 Months \\
\hline Sitting/Lying Time (hours/day) $†$ & $18.6 \pm 1.5$ & $18.3 \pm 1.7$ & $18.1 \pm 1.6$ \\
\hline WeekdaySitting/Lying Time (hours/day) & $18.5 \pm 1.8$ & $18.3 \pm 1.8$ & $18.2 \pm 1.8$ \\
\hline Week-End Sitting/Lying Time(hours/day) & $18.7 \pm 1.9$ & $18.1 \pm 1.9$ & $17.8 \pm 1.9$ \\
\hline Steps/day $\dagger$ & $6139 \pm 2720$ & $6068 \pm 3034$ & $6913 \pm 3073$ \\
\hline Week-End Steps/day & $6059 \pm 3189$ & $5967 \pm 3277$ & $6739 \pm 3109$ \\
\hline Transitions/day† & $54.77 \pm 19.70$ & $49.1 \pm 14.28$ & $49.59 \pm 12.65$ \\
\hline Weekday Transitions/day & $57.16 \pm 22.28$ & $50.36 \pm 15.29$ & $51.26 \pm 16.57$ \\
\hline Week-End Transitions/day & $49.76 \pm 18.04$ & $47.46 \pm 17.93$ & $47.32 \pm 10.55$ \\
\hline Walking Time (hours/day) $†$ & $1.42 \pm .56$ & $1.38 \pm .58$ & $1.54 \pm .57$ \\
\hline Week-End Walking Time (hours/day) & $1.43 \pm .68$ & $1.32 \pm .69$ & $1.53 \pm .61$ \\
\hline Standing Time (hours/day) $\dagger$ & $5.41 \pm 1.51$ & $5.69 \pm 1.66$ & $5.92 \pm 1.62$ \\
\hline Weekday Standing Time (hours/day) & $5.53 \pm 1.80$ & $5.66 \pm 1.81$ & $5.82 \pm 1.76$ \\
\hline Week-End Standing Time (hours/day) & $5.28 \pm 1.87$ & $5.94 \pm 1.93$ & $6.16 \pm 1.92$ \\
\hline
\end{tabular}

*Variables are expressed as mean \pm S.D., $†$ represent values averaged across all days

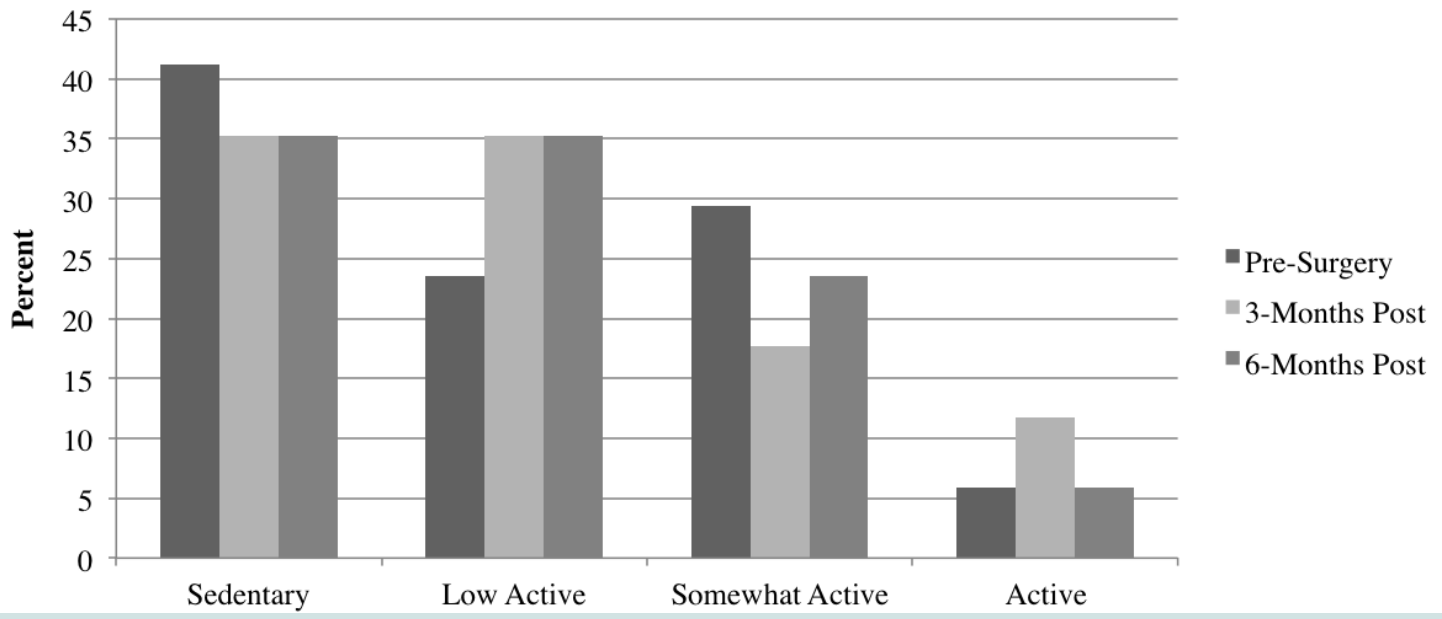

Figure 1: Classification of participant steps/day with established norms over time

to-stand transitions per day did not increase after surgery, a negative correlation between the number of transitions per day and body weight was displayed across all time points (baseline: $\mathrm{r}=-0.49 ; \mathrm{p}=$ 0.044; three-months post; $\mathrm{r}=-0.51 ; \mathrm{p}=0.038$; six-months post; $\mathrm{r}=$ $-0.6 ; \mathrm{p}=0.011)$.

\section{Discussion}

An active lifestyle including structured exercise as well as recreational activities along with active transportation [21] is associated with long-term weight control among individuals who have lost and successfully maintained great amounts of weight over time [22]. This investigation objectively measured time spent sitting/lying per 24 hours, steps as well as sit-to-stand transitions, and confirmed that participants were not sufficiently active while awaiting bariatric surgery. Moreover, steps/day, sitting/lying time per 24 hours, and sit-to-stand transitions did not change at the third or even sixth-month following surgery as compared to baseline values, despite dramatic and significant weight loss. Participants who were already overly sedentary and not meeting PA guidelines failed to become more active and reduce sitting/lying time after bariatric surgery. These findings are consistent with other studies examining the PA habits of bariatric patients before surgery where low levels of PA and elevated levels of sedentary behaviors are common $[6,23]$.

On average, participants did not achieve the active threshold of $\geq$ 10000 steps/day [20] at any time before or after bariatric surgery (Table 2 ), which is comparable to the results of 5784 steps/days obtained 
Citation: Babineau O, Carver TE, Reid RER, Christou NV, Andersen RE. Objectively Monitored Physical Activity and Sitting Time in Bariatric Patients Pre- and Post-Surgery. J Obes Bariatrics. 2015;2(2): 5.

for obese individuals through the National Health and Nutrition Examination Survey (NHANES) [24]. Although no participants reached the active threshold of $\geq 10000$ step/day [20] before surgery, one participant did at month three, and another at month six after surgery, albeit different participants at each time point. This indicates that because no singular participant maintained this recommended level of activity throughout both post-surgical evaluations, that these single active threshold breaks within each assessment period may be more indicative of temporary short-term activity bouts, than the desired change in lifestyle habits required for long-term post-surgery weight loss maintenance. This observation is strengthened by the lack of post-surgical changes in any of the accelerometer derived activity data even when examined for week-days, weekend-days, during the day, and throughout evening leisure time (Table 2).

Alarmingly, nearly one quarter of participants failed to exceed even the 5000 steps/day sedentary threshold before and after surgery. Similarly, the Longitudinal Assessment of Bariatric Surgery Study found that before surgery, $41 \%$ of patients were somewhat active or active, $34 \%$ were low active, and $20 \%$ were sedentary [10] using TudorLocke's step/day activity index [20]. In 2012, King et al. followed up with the aforementioned cohort and found them insufficiently active one-year after surgery [11]. This extreme level of inactivity is associated with multiple health risks including poorer lipid profiles and weight gain [2-5].

Interventions to increase $\mathrm{PA}$ and reduce sedentary time are needed to convey the connection between lifestyle changes and long-term weight loss maintenance for individuals who undergo this surgical procedure [25-27]. A recent study by Bond et al. underlined the importance of considering the intention to exercise in individuals who had undergone the surgery six-months prior. Results indicated that few participants intended to be active on most days of the week and nearly all participants had difficulty achieving the intended levels of PA. These findings suggest that the intention to exercise is not sufficient and that future research should involve tailored interventions to promote and reinforce active living through lifestyle and behaviour change [28]. As walking is one of the easiest and safest ways to establish an exercise program for obese individuals [8], steps/ day may be the optimal prescription immediately following surgery. However, the intensity, duration and type of PA should be considered throughout the entire lifetime in order to obtain optimal long-term results [29]. Additionally, this study suggests that encouraging severely obese individuals to increase the number of their sit-to-stand transitions may be an important behavior to target through such an intervention. There is evidence that breaking up long periods of sitting time is beneficial for avoiding weight gain independent of total daily sedentary time [16], and therefore should be incorporated as another potential area of reducing overall sedentary behaviour, specifically for long-term weight loss maintenance.

We acknowledge that a small sample size may limit the interpretation of these data. However, this study demonstrated that following standard of care, individuals who have undergone bariatric surgery remained inactive even after a major medical procedure designed to reduce weight and improve mobility. Moreover, strength of our study is that it is one of the first investigations to objectively measure the transitions, walking time, and standing time of individuals undergoing bariatric surgery. At the time of data collection, sleeping journals were not used which is why all measures of sitting/lying time (i.e., sedentary time) include sleep time. Studies have reported that in this short time following surgery that few to no changes in sleep length and quality occur [30] leaving us confident that the lack of change in sitting/lying time per 24 hours, sit-to-stand transitions and steps/day from pre-to post-surgery describe a lack in behaviour change as a whole. Moreover, analysis of NHANES data show that $36.4 \%$ of Americans spend $\leq 6$ hours/ 24 hours sleeping and $56.1 \%$ average only 7 to 8 hours/ 24 hours sleeping [31]. Subtracting even the maximum of 8 hours of sleep from this study's recorded sitting/lying time per 24 hours still identifies that the participants of this study were sitting/lying considerably more than that of the national average of 7.7 waking hours [9]. However, future investigations should seek to more accurately quantifying sleep time through sleep journals. A relatively equal number of men and women took part in this study.

\section{Conclusions}

In conclusion, individuals who have undergone bariatric surgery receiving standard care did not change their PA or sitting/lying time per 24 hours in the first three and six-months following surgery. Since both PA and sedentary behavior are believed to independently contribute to energy balance and weight control, combined messages to increase activity levels while simultaneously reducing sedentary time may help bariatric surgery patients achieve optimal weight loss maintenance and health outcomes in the long-term. Further research is needed to determine how health professionals can best intervene with this population in order to develop effective strategies that will promote long-term weight loss maintenance among individuals who have undergone bariatric surgery.

\section{References}

1. Donnelly JE, Blair SN, Jakicic JM, Manore MM, Rankin JW, et al. (2009) American College of Sports Medicine Position Stand. Appropriate physical activity intervention strategies for weight loss and prevention of weight regain for adults. Med Sci Sports Exerc 41: 459-471.

2. Katzmarzyk PT, Church TS, Craig CL, Bouchard C (2009) Sitting time and mortality from all causes, cardiovascular disease, and cancer. Med Sci Sports Exerc 41: 998-1005.

3. Owen N, Healy GN, Matthews CE, Dunstan DW (2010) Too much sitting: the population health science of sedentary behavior. Exerc Sport Sci Rev 38: 105-113

4. Ainsworth BE, Haskell WL, Whitt MC, Irwin ML, Swartz AM, et al. (2000) Compendium of physical activities: an update of activity codes and MET intensities. Med Sci Sports Exerc 32(9 Suppl): S498-S504.

5. Sedentary Behaviour Research Network (2012) Letter to the editor: standardized use of the terms "sedentary" and "sedentary behaviours". Appl Physiol Nutr Metab 37: 540-542.

6. Bond DS, Thomas JG, Unick JL, Raynor HA, Vithiananthan S, et al. (2013) Self-reported and objectively measured sedentary behavior in bariatric surgery candidates. Surg Obes Relat Dis 9: 123-128.

7. Andersen RE, Jakicic JM (2009) Interpreting the physical activity guidelines for health and weight management. J Phys Act Health 6: 651-656.

8. Livhits M, Mercado C, Yermilov I, Parikh JA, Dutson E, et al. (2010) Exercise following bariatric surgery: systematic review. Obes Surg 20: 657-665.

9. Matthews CE, Chen KY, Freedson PS, Buchowski MS, Beech BM, et al. (2008) Amount of time spent in sedentary behaviors in the United States, 2003-2004. Am J Epidemiol 167: 875-881. 
Citation: Babineau O, Carver TE, Reid RER, Christou NV, Andersen RE. Objectively Monitored Physical Activity and Sitting Time in Bariatric Patients Pre- and Post-Surgery. J Obes Bariatrics. 2015;2(2): 5.

ISSN: 2377-9284

10. King WC, Belle SH, Eid GM, Dakin GF, Inabnet WB, et al. (2008) Physical activity levels of patients undergoing bariatric surgery in the Longitudinal Assessment of Bariatric Surgery study. Surg Obes Relat Dis 4: 721-728.

11. King WC, Hsu JY, Belle SH, Courcoulas AP, Eid GM, et al. (2012) Pre- to postoperative changes in physical activity: report from the longitudinal assessment of bariatric surgery-2 (LABS-2). Surg Obes Relat Dis 8: 522-532.

12. Livhits M, Mercado C, Yermilov I, Parikh JA, Dutson E, et al. (2010) Exercise following bariatric surgery: systematic review. Obes Surg 20: 657-665.

13. Jacobi D, Ciangura C, Couet C, Oppert JM (2011) Physical activity and weight loss following bariatric surgery. Obes Rev 12: 366-377.

14. Lau DC, Douketis JD, Morrison KM, Hramiak IM, Sharma AM, et al (2007) 2006 Canadian clinical practice guidelines on the management and prevention of obesity in adults and children [summary]. CMAJ 176: S1-S13.

15. Carver TE, Christou NV, Andersen RE (2013) In vivo precision of the GE iDXA for the assessment of total body composition and fat distribution in severely obese patients. Obesity (Silver Spring) 21: 1367-1369.

16. Healy GN, Dunstan DW, Salmon J, Cerin E, Shaw JE, et al. (2008) Breaks in sedentary time: beneficial associations with metabolic risk. Diabetes Care 31: $661-666$

17. Grant PM, Ryan CG, Tigbe WW, Granat MH (2006) The validation of a novel activity monitor in the measurement of posture and motion during everyday activities. Br J Sports Med 40: 992-997.

18. Ryan CG, Grant PM, Tigbe WW, Granat MH (2006) The validity and reliability of a novel activity monitor as a measure of walking. Br J Sports Med 40: 779784 .

19. Faul F, Erdfelder E, Lang AG, BuchnerA (2007) G*Power 3: a flexible statistical power analysis program for the social, behavioral, and biomedical sciences. Behav Res Methods 39: 175-191.

20. Tudor-Locke C, Bassett DR Jr (2004) How many steps/day are enough? Preliminary pedometer indices for public health. Sports Med 34: 1-8.

21. Sallis JF, Linton L, Kraft MK (2005) The first Active Living Research Conference: growth of a transdisciplinary field. Am J Prev Med 28(2 Suppl 2): 93-95.
22. Catenacci VA, Ogden LG, Stuht J, Phelan S, Wing RR, et al. (2008) Physical activity patterns in the National Weight Control Registry. Obesity (Silver Spring) 16: 153-161.

23. Bond DS, Jakicic JM, Vithiananthan S, Thomas JG, Leahey TM, et al. (2010) Objective quantification of physical activity in bariatric surgery candidates and normal-weight controls. Surg Obes Relat Dis 6: 72-78.

24. Tudor-Locke C, Brashear MM, Johnson WD, Katzmarzyk PT (2010) Accelerometer profiles of physical activity and inactivity in normal weight, overweight, and obese U.S. men and women. Int J Behav Nutr Phys Act 7 : 60.

25. Kozey-Keadle S, Libertine A, Staudenmayer J, Freedson P (2012) The feasibility of reducing and measuring sedentary time among overweight, nonexercising office workers. J Obes 2012: 282303.

26. Josbeno DA, Jakicic JM, Hergenroeder A, Eid GM (2010) Physical activity and physical function changes in obese individuals after gastric bypass surgery. Surg Obes Relat Dis 6: 361-366.

27. King WC, Bond DS (2013) The importance of preoperative and postoperative physical activity counselling in bariatric surgery. Exerc Sport Sci Rev 41: 26-

28. Bond DS, Thomas JG, Ryder BA, Vithiananthan S, Pohl D, et al. (2013) Ecological momentary assessment of the relationship between intention and physical activity behavior in bariatric surgery patients. Int J Behav Med 20: 82-87.

29. Tudor-Locke C, Schuna JM Jr (2012) Steps to preventing type 2 diabetes: exercise, walk more, or sit less? Front Endocrinol (Lausanne) 3: 142.

30. Greenburg DL, Lettieri CJ, Eliasson AH (2009) Effects of surgical weight loss on measures of obstructive sleep apnea: a meta-analysis. Am J Med 122: $535-542$

31. Kant AK, Graubard BI (2014) Association of self-reported sleep duration with eating behaviors of American adults: NHANES 2005-2010. Am J Clin Nutr 100: 938-947.

\section{Acknowledgements}

We appreciate the contributions of all authors to this research paper. Olivier Babineau, Tamara E Carver, and Ryan ER Reid were involved in the literature search, data collection, data analysis and interpretation, the generation of figures and writing the manuscript. Dr. Nicolas Christou aided in the recruitment of participants and provided key information pertaining to this unique population, which helped in the literature search and study design. Dr. Ross E Andersen participated in the study design, data analysis, data interpretation, writing the manuscript. 